\title{
Aneurysms of the Intracranial Segment of the Ophthalmic Artery Trunk: Case Report and Systematic Literature Review
}

\author{
Simone Peschillo ${ }^{1}$ Francesco Biraschi ${ }^{2}$ Francesco Diana $^{1}$ Claudio Colonnese ${ }^{1,3}$ Marco Marenco ${ }^{4}$ \\ Roberto Delfini ${ }^{1}$
}

${ }^{1}$ Department of Neurology and Psychiatry, Endovascular Neurosurgery, University of Rome “Sapienza," Rome, Italy

2 Department of Neurology and Psychiatry, Interventional Neuroradiology, Universita degli Studi di Roma La Sapienza Ringgold Standard Institution, Roma, Lazio, Italy

${ }^{3}$ Department of Neuroradiology, IRCCS INM Neuromed Pozzilli,

Rome, Italy

${ }^{4}$ Department of Sense Organs, Ophthalmology, "Sapienza," University of Rome, Rome, Italy

J Neurol Surg A

\author{
Address for correspondence Simone Peschillo, MD, PhD, Department \\ of Neurology and Psychiatry, University of Rome "Sapienza," Viale del \\ Policlinico 155, Rome 00161, Italy \\ (e-mail: simone.peschillo@gmail.com).
}

\begin{abstract}
Keywords

- systematic review

- aneurysm

- subarachnoid hemorrhage

- ophthalmic artery

Aneurysms arising from the ophthalmic artery trunk (OAT) are very rare, particularly in the artery's intracranial course. The onset of a subarachnoid hemorrhage (SAH) from a ruptured OAT aneurysm in this segment is extremely rare. We present a case and discuss the anatomy, clinical significance, and therapeutic options for an aneurysm at this site. We also retrospectively analyzed the record of a patient with a ruptured aneurysm of the intracranial segment of the OAT and conducted a comprehensive and systematic review of the PubMed and Scopus databases for literature on this pathology. Only one case report of SAH from an aneurysm of the intracranial segment of the OAT was published in the literature. Only in our case was the intracranial OAT segment aneurysm discovered in the acute phase of SAH. Conventional angiography with threedimensional acquisition may help detect aneurysms at this level. Detailed knowledge of the anatomy of the OAT is of paramount importance for both surgical and endovascular approaches. Surgical treatment is complex because of difficulties in accessing the orbital region and the risk of optic nerve and vascular injuries. Endovascular treatment, when feasible, could be a good alternative to reduce the risk of loss of vision related to surgical manipulation.
\end{abstract}

\section{Introduction}

Aneurysms arising from the ophthalmic artery trunk (OAT) are very rare compared with other aneurysms arising from the circle of Willis. OAT aneurysms most commonly present with exophthalmus or progressive visual disturbance, although they may be asymptomatic. ${ }^{1}$ The development of subarachnoid hemorrhage $(\mathrm{SAH})$ as a result of such an aneurysm is an extremely rare event. ${ }^{2}$

received

December 2, 2016

accepted after revision

January 31, 2017

Although intraorbital and intracanalicular OAT aneurysms have been reported, ${ }^{1}$ here we describe a very rare case of a ruptured aneurysm originating from the intracranial segment of the OAT. We discuss the anatomy and clinical significance of an aneurysm at this site, as well as the therapeutic options. We also conducted a systematic review of treatments and outcomes of these rare vascular lesions.

(c) Georg Thieme Verlag KG Stuttgart · New York
DOI https://doi.org/ 10.1055/s-0037-1604268. ISSN 2193-6315. 

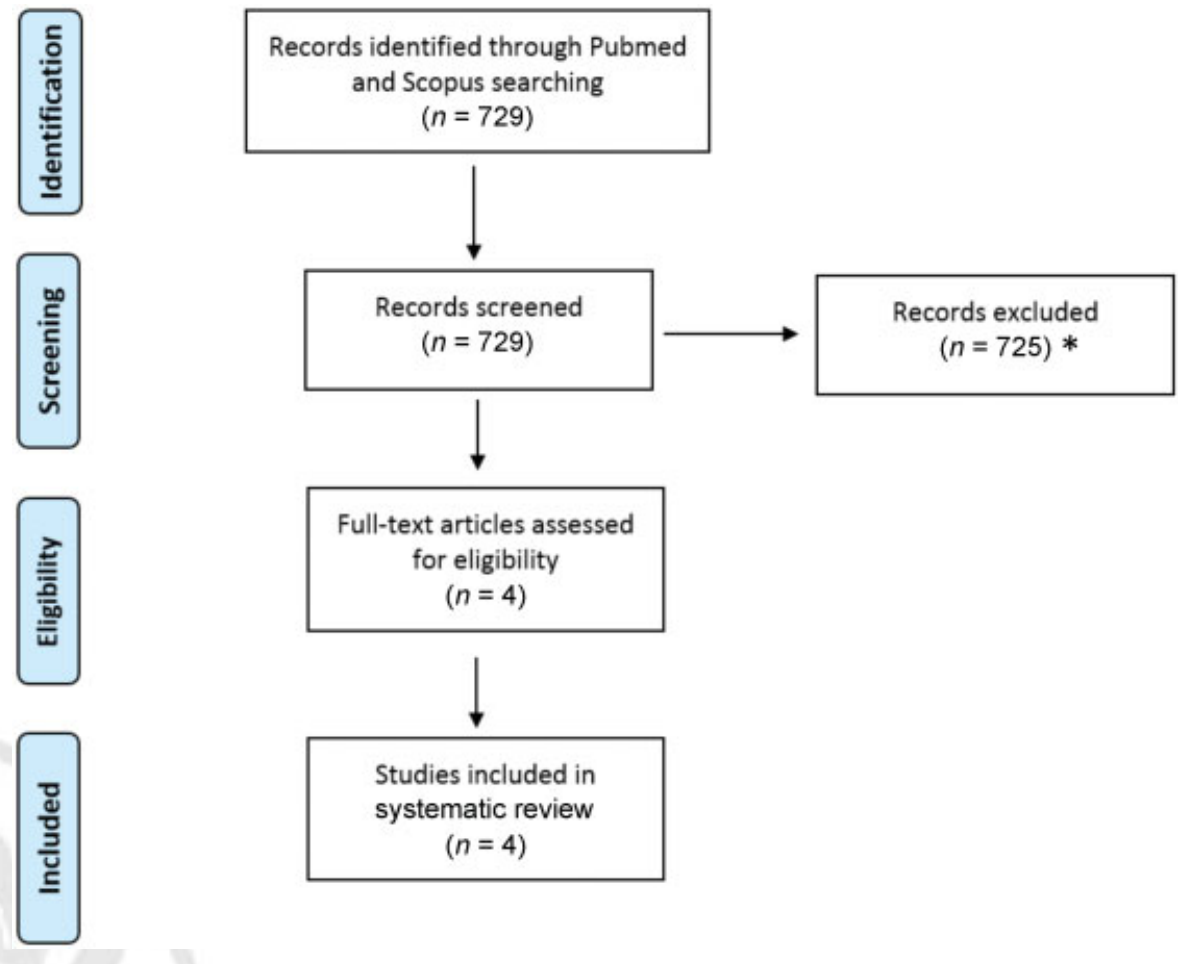

Fig. 1 Preferred Reporting Items for Systematic Reviews and Meta-Analyses flowchart of the systematic review search. *Patients without intracranial segment aneurysms or traumatic aneurysms were excluded.

\section{Material and Methods}

We retrospectively analyzed the record of a patient with a ruptured aneurysm of the intracranial segment of the OAT. We also conducted a comprehensive and systematic literature search of the PubMed and Scopus databases using the keywords ophthalmic artery aneurysms, intracranial segment, and ophthalmic artery trunk. The search was limited to Englishlanguage articles published between January 1950 and May 2016. Two reviewers selected the studies for inclusion.

\section{Results}

Our literature searches initially yielded 729 articles. According to the Preferred Reporting Items for Systematic Review and
Meta-Analyses guidelines for systematic reviews and metaanalyses, ${ }^{3} 725$ articles were excluded because the patients did not have intracranial segment aneurysms of the OAT or had traumatic aneurysms ( - Fig. 1). A total of four studies with four patients met our inclusion criteria. - Table 1 summarizes the published cases of aneurysms of the intracranial segment of the ophthalmic artery treated with surgical techniques.

\section{Illustrative Case}

We describe the case of a 66-year-old woman admitted to our emergency department. She was found on the floor of her house by relatives after having fallen down the stairs. Her medical history included hypertension and alcohol abuse. Physical examination revealed a soft tissue hematoma in the

Table 1 Cases of ophthalmic artery trunk aneurysm reported in the literature ${ }^{\text {a }}$

\begin{tabular}{|l|l|l|l|l|l|l|}
\hline $\begin{array}{l}\text { Case } \\
\text { no. }\end{array}$ & Series & Age/Sex & $\begin{array}{l}\text { Presenting } \\
\text { symptoms }\end{array}$ & Treatment & Outcome & Comment \\
\hline 1 & Jain $^{12}$ & $44 / \mathrm{M}$ & Papilledema & Clipping & Good & - \\
\hline 2 & Sato et al $^{13}$ & $50 / \mathrm{M}$ & SAH & Clipping & Good & $\begin{array}{l}\text { Ruptured anterior communicating } \\
\text { artery aneurysm }\end{array}$ \\
\hline 3 & Kawaguchi et al $^{14}$ & $26 / \mathrm{F}$ & $\begin{array}{l}\text { Episodic } \\
\text { dizziness }\end{array}$ & Clipping & Good & Moyamoya disease \\
\hline 4 & Yanaka et al & & SAH & Clipping & Good & $\begin{array}{l}\text { First DSA failed to localize the source } \\
\text { of the hemorrhage. } \\
\text { Treated } 2 \text { wk after SAH }\end{array}$ \\
\hline 5 & Present case report, 2016 & $66 / \mathrm{F}$ & SAH & None & Death & Low platelet count \\
\hline
\end{tabular}

Abbreviations: DSA, digital subtraction angiography; F, female; M, male; SAH, subarachnoid hemorrhage.

${ }^{a}$ Cases of SAH associated with rupture of the intracranial segment of the ophthalmic artery trunk are shown in bold. 


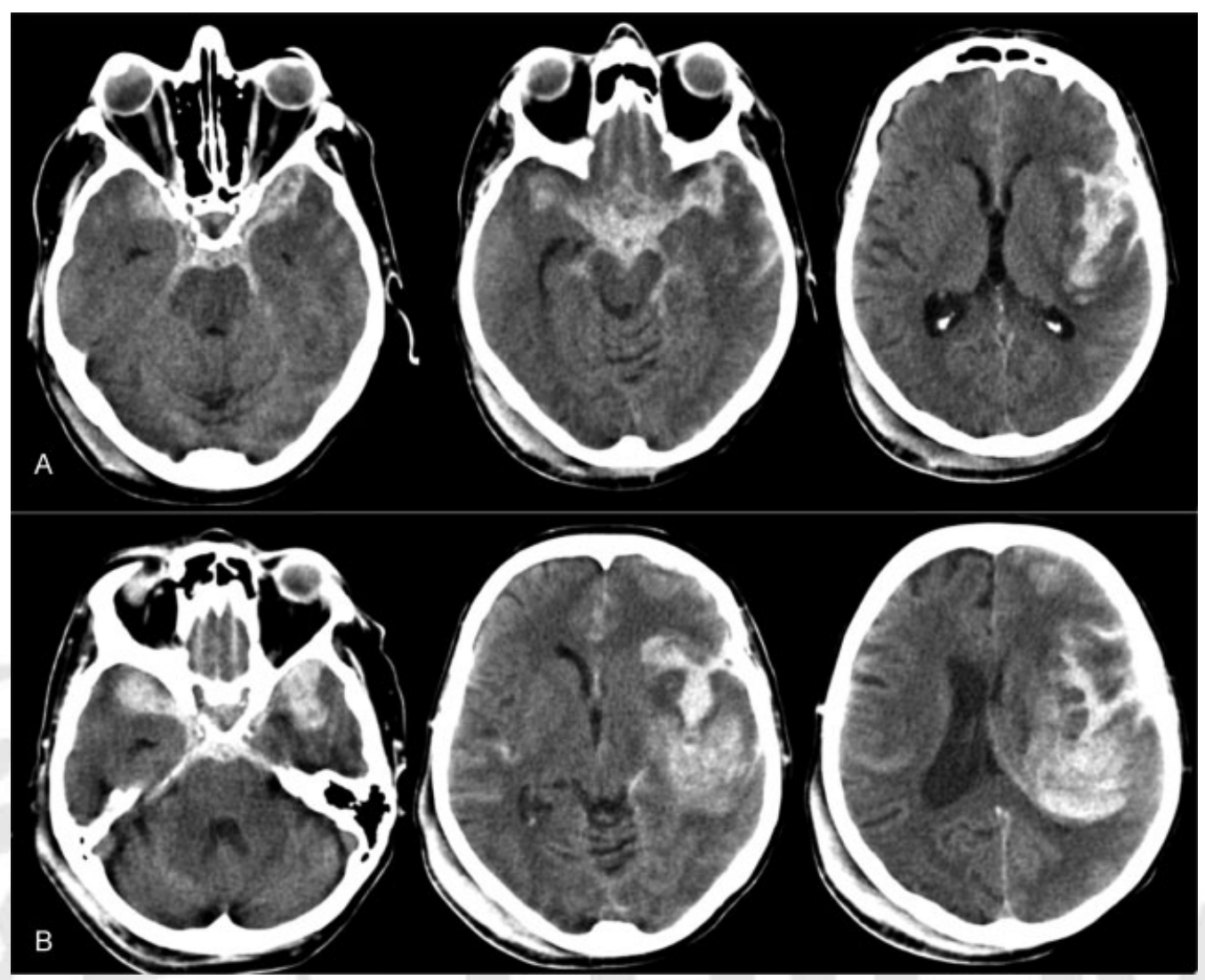

Fig. 2 Computed tomography scans without contrast. (a) Initial scan. (b) Scan after clinical deterioration.

right occipital region. At neurologic examination the patient was drowsy, awakened by speech stimuli, localized the pain with her left arm, and had right-sided hemiplegia (Glasgow Coma Scale [GCS] score 12). Computed tomography (CT) without contrast showed a massive SAH involving the perimesencephalic and basal cisterns and interhemispheric and left sylvian fissures with an associated parenchymal hematoma in the left temporoparietal region ( $\mathbf{- F i g . 2 a}$ ). The CT angiogram did not show an aneurysmatic formation or arteriovenous malformations. Serum blood analysis showed severe thrombocytopenia (platelet count $45 \times 10^{9} / \mathrm{L}$ ), moderate anemia (hemoglobin $9.7 \mathrm{~g} / \mathrm{dL}$ ), slight liver function impairment (aspartate and alanine transaminases 167 and 44 IU/L, respectively), and an international normalized ratio of 1.71 .

During her stay in the emergency department, the patient's neurologic status worsened suddenly (GCS score 5) with compromised consciousness. A new CT without contrast was performed that showed expansion of the left temporoparietal hematoma with a mass effect on the ventricular structures and midline shift (-Fig. 2b). The patient was intubated and a left decompressive craniotomy was performed, after which she was admitted to the angiology suite. Conventional cerebral angiography was performed with three-dimensional (3D) rotational acquisition from the internal carotid artery bilaterally. Oblique images and 3D rotational angiography demonstrated a 1.5-mm saccular aneurysm arising from "angle a" of the right ophthalmic artery (-Fig. 3). Diffuse vasospasm was also observed. Two expert neurointerventionists reviewed the angiographic images and observed no other alterations. Given her severe clinical condition and neurologic impairment, it was decided not to treat the patient but to wait and see. She died the following day.
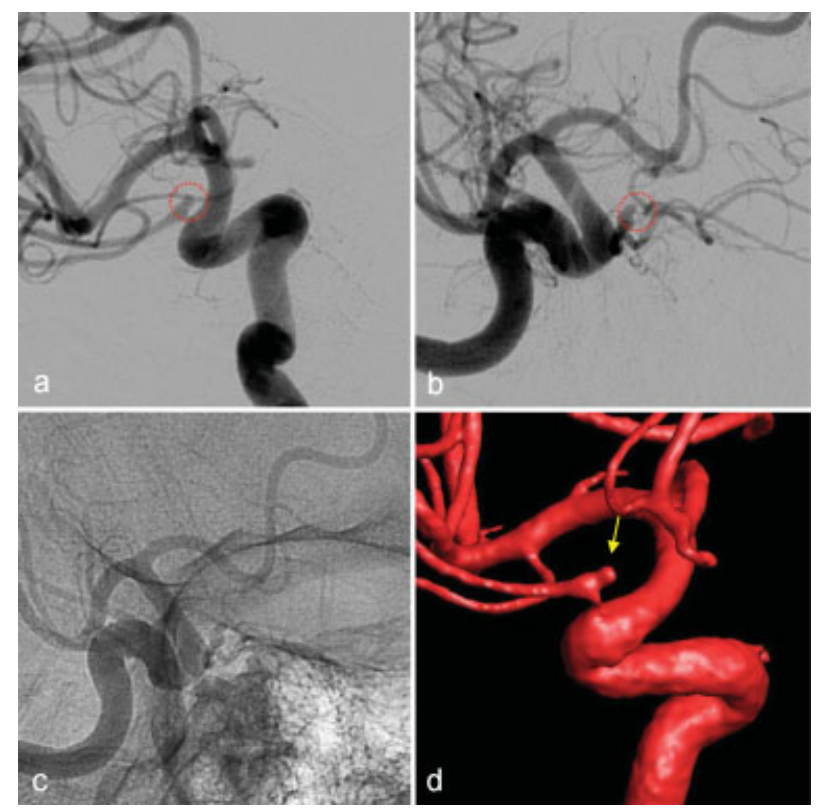

Fig. 3 (a) Small aneurysm of the right intracranial tract (red circle), with (b, c) bleb on the apex of the aneurysmal sac. (d) Three-dimensional reconstruction of the aneurysmal sac (yellow arrow). 


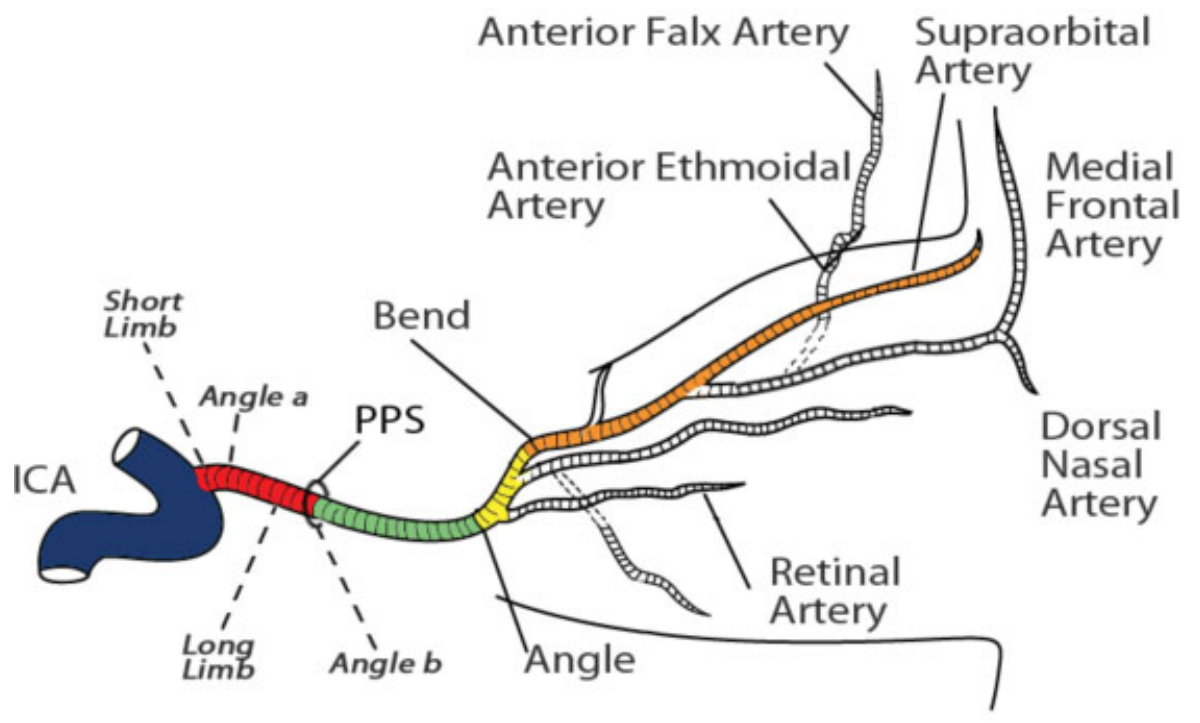

Fig. 4 Diagram illustrating the course and branches of the ophthalmic artery. Intracranial segment is in red, the infraoptic portion of the intraorbital segment in green, the latero-optic portion of the intraorbital segment in yellow, and the supraoptic portion of the intraorbital segment in orange. ICA, internal carotid artery; PPS, point of penetration of dural sheath. (Modified from Shoki Takahashi, Neurovascular Imaging MRI \& Microangiography. London, UK: Springer; 2010.)

\section{Discussion}

The OAT is the first major branch of the internal carotid artery. In 80 to $90 \%$ of patients it has an intradural course because it originates immediately after the distal dural ring, just below the anterior clinoid process, right beneath the optic nerve. ${ }^{1}$ Embryologically, the first segment of the OAT supplies visual structures, the second portion is an anastomotic channel, and the third supplies orbital but nonvisual structures. Following these characteristics, Ogawa et $\mathrm{al}^{4}$ divided the OAT into three portions: intracranial, intracanalicular, and intraorbital (-Fig. 4). The intracranial course of the artery was divided into the short limb, angle "a," long limb, and angle "b" (-Figs. 4 and 5). This small distance (0.5-9.5 mm according to Hayreh and Dass) is of paramount surgical importance. $^{5}$

After that, the OAT passes below the posterior edge of the falciform ligament, pierces the dura mater of the optic nerve, usually inferiorly, and enters the optic canal laterally and alongside the optic nerve. ${ }^{6}$ The division previously mentioned is very important for therapeutic reasons because although there are several anastomoses between the external carotid artery and the OAT within the orbit, the more proximal to the carotid artery the aneurysm, the more often ocular ischemia may occur. ${ }^{7}$ Indeed, the "safe zone" for avoiding any visual impairment is from the supraoptic portion of the intraorbital segment, just after the origin of the central retinal artery ${ }^{8}$ (- Figs. 4 and 5).

OAT aneurysms comprise a subset with a relatively low rate of rupture. ${ }^{1}$ However, aneurysm rupture at this level manifests as SAH with possible fatal consequences.

Only a single published case of $\mathrm{SAH}^{2}$ is associated with rupture of an aneurysm of the intracranial segment of the OAT (-Table 1), and the aneurysm was discovered and treated 2 weeks after the presenting bleed.
It was difficult to identify the source of bleeding in our patient. The spread of the hemorrhage was suggestive of a ruptured aneurysm of the left middle cerebral artery. However, besides widespread vasospasm, digital subtraction angiography (DSA) revealed a small aneurysm $(\sim 1.5 \mathrm{~mm})$ of the right intracranial segment of the OAT with an apical bleb (-Fig. 3), the probable cause of the SAH. Blister aneurysms were excluded, given that both the $\mathrm{CT}$ angiogram on arrival and the DSA, with bilateral 3D reconstructions, 24 hours after the SAH did not show any such aneurysms. 9,10

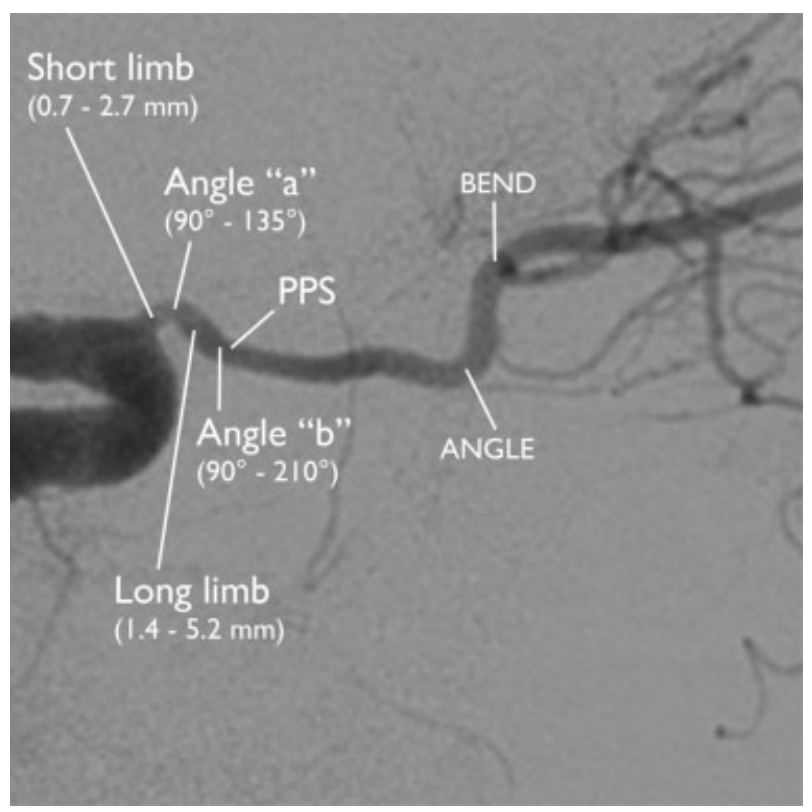

Fig. 5 Angiographic image of the ophthalmic artery. The distinct tracts of the intracranial segment are labeled. 
Such an abundant SAH, associated with two bleeds occurring in a short period, was probably related to the patient's low platelet count and chronic liver disease-induced alterations in clotting factors. The volume of the SAH led us to perform an emergency craniectomy to reduce herniation of the brain.

As far as treatment of aneurysms in this site is concerned, the possibilities include both surgical and endovascular management. Surgery is complicated because access to this area is hampered by the presence of the optic nerve, the dural margin of the optic canal, and the clinoid process. Various techniques have been proposed: extensive removal of the anterior clinoid process, unroofing of the optic canal, mobilization of the ophthalmic artery, and complete circumferential dissection around the internal carotid artery at the level of the dural ring. ${ }^{5}$

Nishio et al proposed a contralateral pterional approach to this type of aneurysm. Considering that the origin of the OAT is often superomedial, a contralateral approach enables the neurosurgeon to avoid the optic nerve. ${ }^{11}$ Loss of vision is an important complication of this type of procedure and is due to manipulation of the OAT and closure of or damage to the small capillaries that run from this tract to the ventral aspect of the optic nerve ${ }^{12}$ or to temporary vascular occlusion that causes sufficient ischemic damage to result in optic neuritis. In all cases, detailed knowledge of the anatomy of the ophthalmic artery is of paramount importance. ${ }^{13}$

In our case, given the patient's clinical conditions and after multidisciplinary discussion between vascular and endovascular neurosurgeons, it was decided to use an endovascular approach to treat the aneurysm and postpone the treatment until the patient's neurologic conditions had improved. Unfortunately, she died the following day, from brain death.

A review of the literature revealed only one other case of aneurysm of the OAT that presented with $\mathrm{SAH}^{2}$ ( - Table 1 ). In this case the authors reported that the chosen management, surgical treatment, had a good outcome. It should be noted, however, that in their case, the aneurysm was treated 2 weeks after the presenting bleed because DSA initially failed to localize the source of the hemorrhage. The other three cases were not ruptured aneurysms of the intracranial segment of the OAT but papilledema (case 1), SAH related to rupture of an aneurysm of the anterior communicating artery (case 2), and episodic dizziness in a patient with a diagnosis of moyamoya disease (case 3 ) (-Table $\mathbf{1}$ ).

\section{Conclusions}

OAT aneurysms usually present with exophthalmus or progressive visual field defects, although they may be asymptomatic, and SAH is a very rare complication. This is the second reported case in the literature of SAH related to the rupture of an aneurysm located in the intracranial segment of the ophthalmic artery but the first one discovered in the acute stage.
Accurate conventional cerebral angiography with 3D rotational acquisition should always be performed to improve evaluation of this anatomical region and detect any aneurysms present. Both surgical and endovascular treatment can be performed. Surgical treatment involves difficulties related to access to the orbital region and the risk of optic nerve injuries. Endovascular treatment, when feasible, could reduce the risk of loss of vision related to surgical manipulation. Detailed knowledge of the anatomy of the ophthalmic artery is crucial in both the diagnostic and therapeutic phases, for surgical as well as endovascular approaches.

\section{References}

1 Piché SL, Haw CS, Redekop GJ, Heran MKS. Rare intracanalicular ophthalmic aneurysm: endovascular treatment and review of the literature. AJNR Am J Neuroradiol 2005;26(08):1929-1931

2 Yanaka K, Matsumaru Y, Kamezaki T, Nose T. Ruptured aneurysm of the ophthalmic artery trunk demonstrated by three-dimensional rotational angiography: case report. Neurosurgery 2002; 51(04):1066-1069; discussion 1069-1070

3 Moher D, Liberati A, Tetzlaff J, Altman DG; PRISMA Group. Preferred reporting items for systematic reviews and meta-analyses: the PRISMA statement. PLoS Med 2009;6(07):e1000097

4 Ogawa A, Tominaga T, Yoshimoto T, Kiyosawa M. Intraorbital ophthalmic artery aneurysm: case report. Neurosurgery 1992; 31(06):1102-1104; discussion 1104

5 Michalinos A, Zogana S, Kotsiomitis E, Mazarakis A, Troupis T. Anatomy of the ophthalmic artery: a review concerning its modern surgical and clinical applications. Anat Res Int 2015; 2015:591961

6 Erdogmus S, Govsa F. Anatomic features of the intracranial and intracanalicular portions of ophthalmic artery: for the surgical procedures. Neurosurg Rev 2006;29(03):213-218

7 Dolenc VV, Carter LP, Spetzler RF. Carotid-ophthalmic aneurysms. In: Carter LP, Spetzler RF, eds. Neurovascular Surgery. New York, NY: McGraw-Hill; 1995:673-686

8 Perrini P, Cardia A, Fraser K, Lanzino G. A microsurgical study of the anatomy and course of the ophthalmic artery and its possibly dangerous anastomoses. J Neurosurg 2007;106(01):142-150

9 Peschillo S, Miscusi M, Caporlingua A, et al. Blister-like aneurysms in atypical locations: a single-center experience and comprehensive literature review. World Neurosurg 2015;84(04):1070-1079

10 Peschillo S, Cannizzaro D, Caporlingua A, Missori P. A systematic review and meta-analysis of treatment and outcome of blisterlike aneurysms. AJNR Am J Neuroradiol 2016;37(05):856-861

11 Nishio S, Matsushima T, Fukui M, Sawada K, Kitamura K. Microsurgical anatomy around the origin of the ophthalmic artery with reference to contralateral pterional surgical approach to the carotid-ophthalmic aneurysm. Acta Neurochir (Wien) 1985;76 (3-4):82-89

12 Jain KK. Saccular aneurysm of the ophthalmic artery. Am J Ophthalmol 1970;69(06):997-998

13 Sato S, Suga S, Ohira T, Takayama H, Kawase T. Aneurysm of the ophthalmic artery trunk. Acta Neurochir (Wien) 1999;141(03): 321-322

14 Kawaguchi S, Sakaki T, Okuno S, Uchiyama Y, Nishioka T. Peripheral ophthalmic artery aneurysm. Report of two cases. J Neurosurg 2001;94(05):822-825 